\title{
Recognition of Antibiotic Resistance in Spontaneous Bacterial Peritonitis Caused by Escherichia coli in Liver Cirrhotic Patients in Civil Hospital Karachi
}

\author{
Pooja D. Kirplani ${ }^{1}$, Laila Tul Qadar ${ }^{2}$, Rohan Kumar Ochani ${ }^{2}$, Zahid Ali Memon ${ }^{3}$, Syeda Anjala Tahir ${ }^{4}$, \\ Khalid Imran ${ }^{5}$, Naresh Kumar Seetlani ${ }^{6}$, Amanullah Abbasi ${ }^{2}$, Mahaish Kumar ${ }^{7}$, Piyar Ali ${ }^{4}$

\begin{abstract}
1. Internal Medicine, Civil Hospital, Karachi, PAK 2. Internal Medicine, Dow University of Health Sciences, Karachi, PAK 3. Surgery, Civil Hospital Karachi, Dow University of Health Sciences, Karachi, PAK 4. Internal Medicine, Civil Hospital Karachi, Dow University of Health Sciences, Karachi, PAK 5. Internal Medicine: Gastroenterology, Civil Hospital Karachi, Dow University of Health Sciences, Karachi, PAK 6. Internal Medicine: Infectious Disease, Civil Hospital Karachi, Dow University of Health Sciences, Karachi, PAK 7. Internal Medicine, Aga Khan University Hospital, Karachi, PAK
\end{abstract}

Corresponding author: Rohan Kumar Ochani, rohanochani@gmail.com

\section{Abstract \\ Introduction}

Spontaneous bacterial peritonitis (SBP) is the most common life-threatening infection in patients with ascites due to liver cirrhosis. The infection is most commonly caused by the bacterium Escherichia coli, commonly referred to as E. coli. Over the past few years, the incidence of antimicrobial resistance against $\mathrm{E}$. coli has risen drastically, leading to increased morbidity and mortality.

\section{Methods}

This cross-sectional study was conducted to determine the pattern of resistance using variations of antibiotics against E. coli, to prevent its empirical usage and initiate an appropriate target antibiotic therapy. The data were collected from May 2017 to October 2017 and included a total of 184 patients. The patients had previously been diagnosed with chronic liver disease and had presented with E. coli-induced SBP in the medicine wards at Civil Hospital, Karachi, which is the largest tertiary care hospital in the city. All participants underwent diagnostic paracentesis, and the ascitic fluid samples were sent to labs for culture and sensitivity to antibiotics.

\section{Results}

The sample population consisted of 184 participants, of which two-thirds $(63.6 \% ; n=117 / 184)$ of the population consisted of males. The mean age of the participants was $47.6 \pm 10.7$ years. More than half of the patients had hepatitis C $(54.9 \%$; $=101 / 184)$ while the remaining were diagnosed with hepatitis B $(45.1 \%$; $\mathrm{n}=83 / 184)$. The ascitic fluid showed varying percentages of resistance for drugs, with no resistance to imipenem and meropenem while ciprofloxacin showed the highest resistance in eradicating the bacterium, E. coli. Additionally, a statistical correlation was tested between drug resistance and factors like age, gender, duration of liver disease, and duration of ascites. Ciprofloxacin and tetracycline showed a positive correlation between the resistance of these drugs and the age, gender, and duration of chronic liver disease in the participants while trimethoprim/sulfamethoxazole, amoxicillin/clavulanic acid, and piperacillin/tazobactam showed a positive association with the duration of ascites.

\section{Conclusion}

A rapid diligent intervention of cirrhotic patients with complicated ascites is crucial to alleviate patient mortality. Due to the rising bacterial resistance, primarily, epidemiological patterns should be assessed and analyzed in our regional hospitals, and then, antibiotics should be prescribed meticulously.

Categories: Gastroenterology, Infectious Disease, Other

Keywords: escherichia coli (e. coli), antibiotic-resistance, spontaneous bacterial peritonitis, ascites, end stage liver disease, broad-spectrum antibiotics, paracentesis

\section{Introduction}

Ascites is the most common complication in patients with liver cirrhosis or end-stage liver disease (ESLD), consisting of more than $50 \%$ of the cases [1]. Cirrhotic patients, due to ascites, develop spontaneous bacterial peritonitis (SBP), which is the most common life-threatening infection of ascitic fluid. Its incidence ranges from $10 \%$ to $30 \%$ [2] and has several variable etiologies, with the spreading of bacteria from the gut to 
the lymph nodes being the most common underlying pathogenesis [3].

Escherichia coli (E. coli), an intestinal gram-negative bacterium, is known to be a significant cause of SBP, with non-enterococcus streptococci being the second [4]. According to a study conducted in 2011, the frequency of E. coli isolated from ascitic fluid in SBP was $66.6 \%$, which went up by three times from $22 \%$ in $2010[5-6]$.

SBP has a varying clinical presentation, ranging from being asymptomatic to developing symptoms like abdominal pain, fever, nausea, and diarrhea. The disease can also lead to deteriorating symptoms, such as increased confusion and encephalopathy [7]. The diagnosis of SBP is classically made by looking at the ascitic fluid's neutrophil count of more than or equal to $250 \mathrm{cells} / \mathrm{mm}^{3}$ in the presence of a positive culture [8]. However, the culture usually takes four to five days to confirm the results, leading to a rise in empirical treatment and delayed targeted therapy. This has caused the mortality rate of SBP to rise drastically despite its early diagnosis and prompt management [9].

SBP has a staggering mortality rate of $40 \%-70 \%$ in cirrhotic patients and further rises to $80 \%$ for patients who develop septic shock secondary to the infection [10]. Additionally, the constant neglect in starting definitive therapy has led the mortality rate to rise from $10 \%$ to $46 \%$ [11-12]. Therefore, there is a crucial need to recognize the antimicrobial resistance pattern of $\mathrm{E}$. coli so that the targeted antibiotic therapy can be started more readily. Hence, we conducted this study to determine the frequency of patterns of antibiotic resistance in cases of SBP caused by E. coli in patients with liver cirrhosis presented in a tertiary care hospital of Karachi, Pakistan.

The practice of empirical treatment has increased the antimicrobial resistance to certain drugs, making the eradication of the organisms causing SBP a major challenge. A review by Sola et al. showed the rising failure of conventional treatment options, such as third-generation cephalosporin (TGC) [13], although local studies have shown no such problems [14].

\section{Materials And Methods}

This cross-sectional study was carried out in Karachi, Pakistan, from May 2017 to October 2017. The sample population consisted of male and female patients aged 12 years and above. The patients presented to Civil Hospital Karachi and only patients with previously diagnosed liver cirrhosis were chosen, irrespective of their precipitating etiology. They had a Child-Pugh score of B or C and currently presented with the complaint of painful abdominal distention with or without fever. A non-probability consecutive technique was used to calculate the sample size, keeping a confidence level of $95 \%$ and a margin of error of $7 \%$.

The study only included subjects who presented with symptoms of SBP, which are painful abdominal distention in the presence or absence of fever and were diagnosed with abdominal ascites on ultrasound (U/S) abdomen at the time of presentation. Patients only diagnosed with community-acquired SBP were included. Informed verbal consent was also taken from all participants before enrolling them in the study.

The diagnosis of chronic liver disease (CLD) was made based on clinical examination and radiological evidence. Participants with a precipitating etiology other than CLD, such as nephrotic syndrome and congestive heart failure, were excluded. Moreover, patients who were diagnosed with SBP and were either on antibiotics or had received antibiotics in the past 10 days, as well as patients who had a poly-microbial growth in the ascitic fluid culture, were also excluded.

According to the symptoms and the patient's significant history of liver disease, a high index of suspicion for ascites complicated with SBP was kept in mind. To confirm the diagnosis of ascites, patients underwent a departmental U/S, and then they were sent for paracentesis. The procedure was performed by a qualified postgraduate trainee doctor who followed standard precautionary guidelines and used all the aseptic techniques.

The procedure was as follows: a total of $20 \mathrm{ml}$ ascitic fluid was collected from each patient for the detailed report (DR) and culture and sensitivity (CS). Ten ml of ascitic fluid was placed in blood culture bottles at the bedside using an aseptic technique. Three $\mathrm{ml}$ of the sample was directly cultured on two separate blood agar plates and a chocolate agar plate, after centrifuging and incubating it at $37^{\circ} \mathrm{C}$. The culture plates were monitored daily for the next two days to identify any significant growth by a qualified microbiologist of a tertiary care hospital laboratory. Resistance was monitored against various antibiotics: ampicillin/sulbactam, trimethoprim/ sulfamethoxazole (co-trimoxazole), piperacillin, ciprofloxacin, tetracycline (doxycycline), amikacin, ofloxacin, ampicillin, amoxicillin/ clavulanic acid, ceftriaxone, piperacillin/tazobactam, imipenem, and meropenem.

The final results of the ascitic routine examination were made available within a day while the culture and sensitivity report was made available after one week. Until then, the patients were treated empirically with broad-spectrum antibiotics, and the response of the therapy was evaluated using the patients' symptoms. 


\section{Cureus}

Data were entered and analyzed using IBM SPSS Statistics v. 22.0 (Armonk, NY, US). Descriptive statistics were used to report frequencies and proportions for the categorical responses. Variables such as gender, age, duration of CLD, and duration of ascites were stratified to see the effect of these variables on E. colimediated resistance against the aforementioned drugs. T-test was applied for post-stratification, and a pvalue of $\leqslant 0.05$ was taken as significant.

\section{Results}

The sample population consisted of 184 patients, of which 117 (63.6\%) were males and 67 (36.4\%) were females, with a mean age of $47.6 \pm 10.7$ years. Of the 184 participants, about half of them were diagnosed with hepatitis B (45.1\%; $n=83 / 184)$ and more than half had hepatitis C (54.9\%; $n=101 / 184)$. Figure 1 shows the age distribution of the patients.

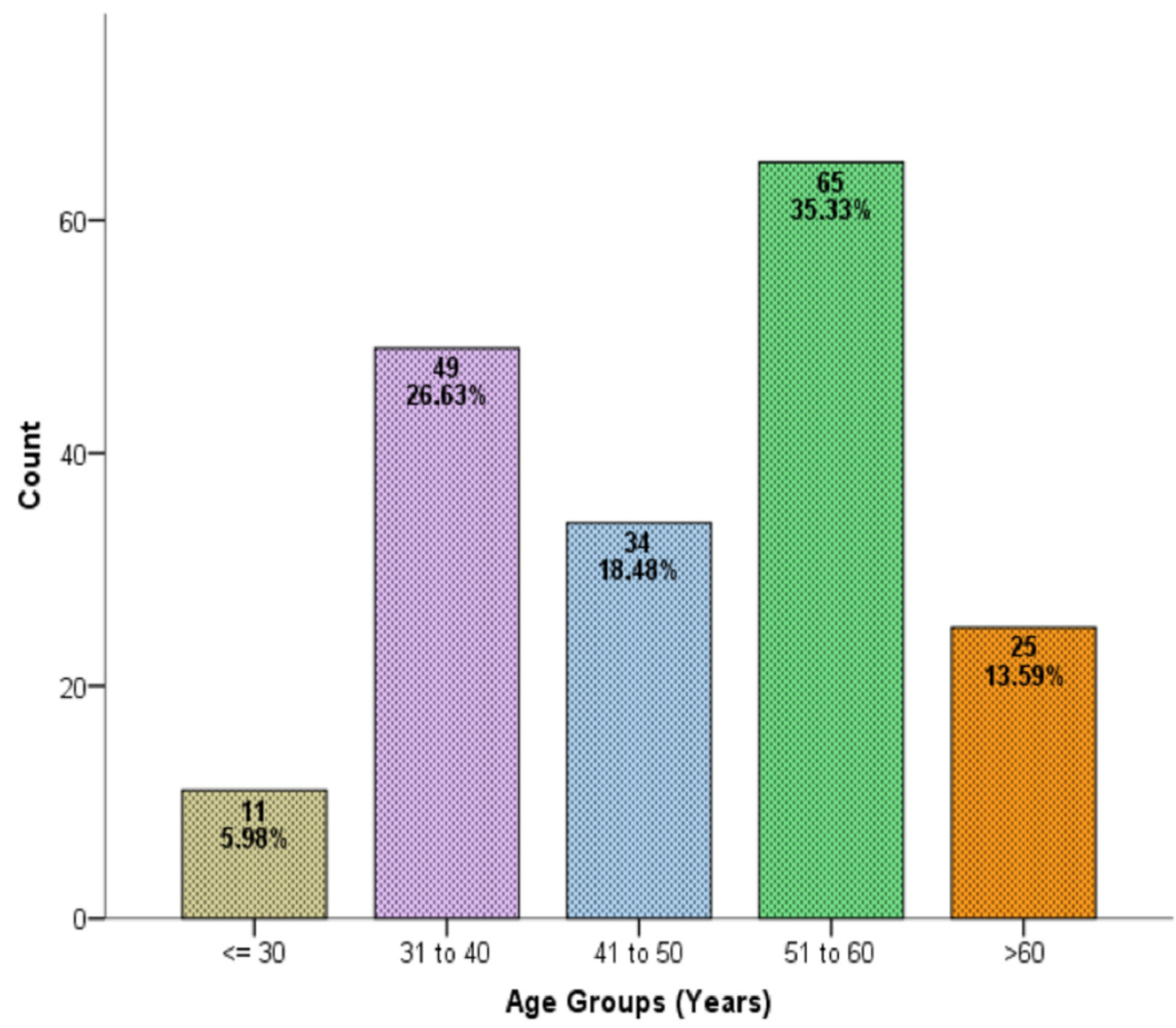

\section{FIGURE 1: Age distribution of the patients}

A confirmed diagnosis was made utilizing the ascitic fluid detailed report while the organism was identified by performing ascitic fluid culture and sensitivity. The most common organism on culture was E. coli; hence, the drugs used to treat SBP due to E. coli had various resistance patterns, which is shown in Table 1.

Amongst all the drugs, the highest resistance was seen with ciprofloxacin and ampicillin, accounting for $38.6 \%$ and $37.5 \%$, respectively, while no resistance was seen with the use of imipenem and meropenem. 


\section{Cureus}

\begin{tabular}{|c|c|c|}
\hline \multirow{2}{*}{ ANTIBIOTICS } & \multicolumn{2}{|c|}{ RESISTANCE } \\
\hline & $\mathrm{N} / 184$ & $\%$ \\
\hline Ampicillin/Sulbactam & 5 & $2.7 \%$ \\
\hline Trimethoprim/Sulfamethoxazole & 20 & $10.9 \%$ \\
\hline Piperacillin & 1 & $0.5 \%$ \\
\hline Ciprofloxacin & 71 & $38.6 \%$ \\
\hline Tetracycline & 52 & $28.3 \%$ \\
\hline Amikacin & 6 & $3.3 \%$ \\
\hline Ofloxacin & 24 & $13 \%$ \\
\hline Ampicillin & 69 & $37.5 \%$ \\
\hline Amoxicillin/Clavulanic acid & 33 & $17.9 \%$ \\
\hline Ceftriaxone & 59 & $32.1 \%$ \\
\hline Cefotaxime & 22 & $12 \%$ \\
\hline Piperacillin/Tazobactam & 12 & $6.5 \%$ \\
\hline Imipenem & 0 & $0 \%$ \\
\hline Meropenem & 0 & $0 \%$ \\
\hline
\end{tabular}

TABLE 1: Frequency of antibiotic resistance in spontaneous bacterial peritonitis caused by $\mathrm{E}$. coli in patients with liver cirrhosis

Table 2 shows the frequency of antibiotic resistance in SBP caused by E. coli and its association with age groups. Ciprofloxacin, tetracycline, and ampicillin expressed the highest resistance in participants less than 50 years of age. Of which, ciprofloxacin $(\mathrm{p}=0.023)$ and tetracycline $(\mathrm{p}=0.035)$ showed a significant correlation between the resistance of these certain drugs and age groups. 


\section{Cureus}

\begin{tabular}{|c|c|c|c|}
\hline \multirow{3}{*}{ ANTIBIOTICS } & \multicolumn{2}{|l|}{ RESISIANCE } & \multirow{3}{*}{ P-Value } \\
\hline & Age $\leq 50$ Years $(\mathrm{N}=94)$ & Age $>50$ Years $(\mathrm{N}=90)$ & \\
\hline & $\mathrm{N}(\%)$ & $\mathrm{N}(\%)$ & \\
\hline Ampicillin/Sulbactam & $4(4.3 \%)$ & $1(1.1 \%)$ & 0.190 \\
\hline Trimethoprim/Sulfamethoxazole & $7(7.4 \%)$ & $13(14.4 \%)$ & 0.127 \\
\hline Piperacillin & $1(1.1 \%)$ & $0(0 \%)$ & 0.085 \\
\hline Ciprofloxacin & $44(46.8 \%)$ & $27(30 \%)$ & 0.023 \\
\hline Tetracycline & $33(35.1 \%)$ & $19(21.1 \%)$ & 0.035 \\
\hline Amikacin & $3(3.2 \%)$ & $3(3.3 \%)$ & 0.99 \\
\hline Ofloxacin & $11(11.7 \%)$ & $13(14.4 \%)$ & 0.581 \\
\hline Ampicillin & $33(35.1 \%)$ & $36(40 \%)$ & 0.493 \\
\hline Amoxicillin/Clavulanic acid & $12(12.8 \%)$ & $21(23.3 \%)$ & 0.062 \\
\hline Ceftriaxone & $28(29.8 \%)$ & $31(34.4 \%)$ & 0.499 \\
\hline Cefotaxime & $10(10.6 \%)$ & $12(13.3 \%)$ & 0.573 \\
\hline Piperacillin//azobactam & $4(4.3 \%)$ & $8(8.9 \%)$ & 0.203 \\
\hline Imipenem & $0(0 \%)$ & $0(0 \%)$ & NA \\
\hline Meropenem & $0(0 \%)$ & $0(0 \%)$ & NA \\
\hline
\end{tabular}

TABLE 2: Frequency of antibiotic resistance in spontaneous bacterial peritonitis caused by E. coli in patients with liver cirrhosis by age groups

Table 3 shows the frequency of antibiotic resistance in SBP caused by E. coli and its association with gender. The resistance of ciprofloxacin and ampicillin were highest in the male gender while females had the highest resistance to ampicillin and ceftriaxone. Ciprofloxacin $(\mathrm{p}=0.007)$, tetracycline $(\mathrm{p}=0.002)$, and piperacillin/tazobactam $(\mathrm{p}=0.007)$ showed a significant correlation between antibiotic resistance and gender. 


\section{Cureus}

\begin{tabular}{|c|c|c|c|}
\hline \multirow{3}{*}{ ANTIBIOTICS } & \multicolumn{2}{|l|}{ RESISTANCE } & \multirow{2}{*}{ P-Value } \\
\hline & Male $n=117$ & Female $\mathrm{n}=67$ & \\
\hline & $\mathrm{n}(\%)$ & $\mathrm{n}(\%)$ & \\
\hline Ampicillin/Sulbactam & $3(2.6 \%)$ & $2(3 \%)$ & 0.866 \\
\hline Trimethoprim/Sulfamethoxazole & $13(11.1 \%)$ & $7(10.4 \%)$ & 0.889 \\
\hline Piperacillin & $0(0 \%)$ & $1(1.5 \%)$ & 0.364 \\
\hline Ciprofloxacin & $54(46.2 \%)$ & $17(25.4 \%)$ & 0.007 \\
\hline Tetracycline & $28(41.8 \%)$ & $24(20.5 \%)$ & 0.002 \\
\hline Amikacin & $6(5.1 \%)$ & $0(0 \%)$ & 0.059 \\
\hline Ofloxacin & $18(15.4 \%)$ & $6(9 \%)$ & 0.213 \\
\hline Ampicillin & $42(35.9 \%)$ & $27(40.3 \%)$ & 0.533 \\
\hline Amoxicillin/Clavulanic acid & $22(18.8 \%)$ & $11(16.4 \%)$ & 0.685 \\
\hline Ceftriaxone & $38(32.5 \%)$ & $21(31.3 \%)$ & 0.874 \\
\hline Cefotaxime & $17(14.5 \%)$ & $5(7.5 \%)$ & 0.155 \\
\hline Piperacillin/Tazobactam & $12(10.3 \%)$ & $0(0 \%)$ & 0.007 \\
\hline Imipenem & $0(0 \%)$ & $0(0 \%)$ & NA \\
\hline Meropenem & $0(0 \%)$ & $0(0 \%)$ & NA \\
\hline
\end{tabular}

TABLE 3: Frequency of antibiotic resistance in spontaneous bacterial peritonitis caused by E. coli in patients with liver cirrhosis by gender

Table 4 shows the frequency of antibiotic resistance in SBP caused by E. coli and its association with the duration of CLD. Several antibiotics had a positive association with the duration of CLD, where ciprofloxacin and ceftriaxone had the highest resistance in participants with a duration of less than four years. 


\section{Cureus}

\begin{tabular}{|c|c|c|c|}
\hline \multirow{3}{*}{ ANTIBIOTICS } & \multicolumn{2}{|l|}{ RESISTANCE } & \multirow{2}{*}{$\begin{array}{l}\text { P- } \\
\text { Value }\end{array}$} \\
\hline & $\begin{array}{l}\text { Duration of chronic liver disease } \leq 4 \text { Years } \\
n=128\end{array}$ & $\begin{array}{l}\text { Duration of chronic liver disease }>4 \text { Years } \\
n=56\end{array}$ & \\
\hline & $\mathrm{n}(\%)$ & $\mathrm{n}(\%)$ & \\
\hline Ampicillin/Sulbactam & $4(3.1 \%)$ & $1(1.8 \%)$ & 0.607 \\
\hline Trimethoprim/Sulfamethoxazole & $18(14.1 \%)$ & $2(3.6 \%)$ & 0.035 \\
\hline Piperacillin & $1(0.8 \%)$ & $0(0 \%)$ & 0.507 \\
\hline Ciprofloxacın & $59(46.1 \%)$ & $12(21.4 \%)$ & 0.002 \\
\hline Tetracycline & $27(21.1 \%)$ & $25(44.6 \%)$ & 0.001 \\
\hline Amikacin & $5(3.9 \%)$ & $1(1.8 \%)$ & 0.456 \\
\hline Ofloxacin & $6(4.7 \%)$ & 18(32.1\%) & 0.0005 \\
\hline Ampicillin & $39(30.5 \%)$ & $30(53.6 \%)$ & 0.003 \\
\hline Amoxicillin/Clavulanic acid & $31(24.2 \%)$ & $2(3.6 \%)$ & 0.001 \\
\hline Ceftriaxone & $47(36.7 \%)$ & $12(21.4 \%)$ & 0.041 \\
\hline Cefotaxime & $10(7.8 \%)$ & $12(21.4 \%)$ & 0.009 \\
\hline Piperacillin/Tazobactam & $7(5.5 \%)$ & $5(8.9 \%)$ & 0.382 \\
\hline Imipenem & 0 & 0 & NA \\
\hline Meropenem & 0 & 0 & NA \\
\hline
\end{tabular}

TABLE 4: Frequency of antibiotic resistance in spontaneous bacterial peritonitis caused by $\mathrm{E}$. coli in patients with liver cirrhosis by duration of chronic liver disease

Table 5 shows the frequency of antibiotic resistance in SBP caused by E. coli and its relation to the duration of ascites. A high resistant pattern was seen by ciprofloxacin, tetracycline, ceftriaxone, and ampicillin in both the groups, although a significant correlation was found between the drugs trimethoprim/sulfamethoxazole (TMP-SMX), amoxicillin/clavulanic acid, and piperacillin/tazobactam and the duration of ascites. 


\section{Cureus}

\begin{tabular}{|c|c|c|c|}
\hline \multirow{3}{*}{ ANTIBIOTICS } & \multicolumn{2}{|l|}{ RESISTANCE } & \multirow{3}{*}{ P-Value } \\
\hline & Duration of Ascites $\leq 24 \mathrm{~h} \mathrm{n}=162$ & Duration of Ascites $>24 \mathrm{~h} \mathrm{n}=22$ & \\
\hline & $\mathrm{n}(\%)$ & $\mathrm{n}(\%)$ & \\
\hline Ampicillin/Sulbactam & $3(1.9 \%)$ & $2(9.1 \%)$ & 0.109 \\
\hline Trimethoprim/Sulfamethoxazole & $13(8 \%)$ & $7(31.8 \%)$ & 0.004 \\
\hline Piperacillin & $1(0.6 \%)$ & $0(0 \%)$ & 0.988 \\
\hline Ciprofloxacin & $61(37.7 \%)$ & $10(45.5 \%)$ & 0.481 \\
\hline Tetracycline & $42(25.9 \%)$ & $10(45.5 \%)$ & 0.056 \\
\hline Amikacin & $4(2.5 \%)$ & $2(9.1 \%)$ & 0.101 \\
\hline Ofloxacin & $24(14.8 \%)$ & $0(0 \%)$ & 0.053 \\
\hline Ampicillin & $62(38.3 \%)$ & $7(31.8 \%)$ & 0.557 \\
\hline Amoxicillin/Clavulanic acid & $25(15.4 \%)$ & $8(36.4 \%)$ & 0.016 \\
\hline Ceftriaxone & $49(30.2 \%)$ & $10(45.5 \%)$ & 0.152 \\
\hline Cefotaxime & $17(10.5 \%)$ & $5(22.7 \%)$ & 0.097 \\
\hline Piperacillin/Tazobactam & $5(3.1 \%)$ & $7(31.8 \%)$ & 0.005 \\
\hline Imipenem & 0 & 0 & NA \\
\hline Meropenem & 0 & 0 & NA \\
\hline
\end{tabular}

TABLE 5: Frequency of antibiotic resistance in spontaneous bacterial peritonitis caused by E. coli in patients with liver cirrhosis by duration of ascites

\section{Discussion}

ESLD carries a global burden for health care, with an estimated 1.2 million mortalities [15-16]. Patients with ESLD pose a weaker immune system and, therefore, have increased exposure to bacterial attacks, sepsis, and death. Bacterial infections in patients with liver cirrhosis are not only more frequent but also are responsible for the patient's deteriorating condition.

SBP, with a prevalence of $8 \%-27 \%$ in patients with cirrhotic ascites, remains amongst the most significant complication in these patients [17]. Most SBP cases (70\%) are caused by gram-negative bacteria, most commonly due to E. coli. As seen similarly to our results, using the mechanism of enterobacteria translocating from the intestinal tract [18].

Past literature has shown a four-fold increase in the mortality of patients reaching almost $58 \%$ in one month and 63\% in 12 months [19]. One of the significant reasons for such high mortality is the rising antimicrobial resistance to antibiotics, including cephalosporin; especially, cefotaxime, fluoroquinolones, ampicillin/sulbactam, and aminoglycosides [20]. The dire consequences require prompt diagnosis and appropriate treatment of the infection when managing patients with liver cirrhosis [21].

Moreover, our study showed that $55 \%$ of the participants had hepatitis $\mathrm{C}$ as the identifiable cause of their liver disease. This was in accordance with a study done by Toshikuni $\mathrm{N}$ et al., where $67 \%-91 \%$ of the participants were diagnosed with hepatitis $\mathrm{C}$ as the primary etiology of cirrhosis [22].

Furthermore, our results showed a significantly elevated (38.6\%) resistance to ciprofloxacin against the eradication of E. coli. This is in congruence with a study conducted by Ortiz et al. where E. coli showed resistant to the use of fluoroquinolones and their failure as a successful prophylactic agent against SBP [23]. Similarly, another study done in Spain enumerated an increased probability in the occurrence of SBP in patients who took fluoroquinolones as a prophylactic agent, concluding intravenous ceftriaxone as a more efficacious drug for SBP prophylaxis [24]. The reasons demonstrated behind the failure of oral fluoroquinolones were primarily due to its slow onset and changing the epidemiology of infections [24]. Although, contrary to our results, a study done in 2017 showed that the intake of oral fluoroquinolones was successful in preventing and treating SBP [25]. 
Conversely, studies conducted in Italy and France manifested reduced efficacy with the use of TGC for SBP with a failure rate of $41 \%$ and $57 \%$, respectively [26-27]. However, our study showed a bacterial resistance of $32.1 \%$, which was far less than the proportion of resistance in the west.

In the present study, sensitivity to piperacillin-tazobactam, amikacin, ampicillin/sulbactam, imipenem, and meropenem was better with imipenem and meropenem being $0 \%$, showed by Lutz P et al. as well [28]. Hence, several studies have recommended their usage as initial treatment as well as in the prophylaxis of SBP caused by gram-negative organisms [28-29].

In the year 2000 , only $1.2 \%$ of bacteria were resistant to TGCs. Today, unfortunately, due to the rising antibiotic resistance, several studies have shown a rise in multidrug-resistant (MDR) bacterial infections in cirrhotic patients. A study conducted in 2012 discovered a swift rise from $10 \%$ to $23 \%$ in the prevalence of MDR bacterial infections between 1998 and 2011 [17], therefore, making it crucial for all medical professionals to meticulously choose antibiotics and ultimately reducing the aftermaths of the increasing resistant pattern.

Having said that, our study had some limitations that need to be considered. Since only patients from a single tertiary-care hospital were specifically recruited in the study, the results of this study cannot be extrapolated to other hospitals. Therefore, further studies should be conducted in order to determine the generalizability of the results. Additionally, we excluded patients who were diagnosed with nosocomial SBP. Nevertheless, the findings of our study are vital in assessing the role of antibiotic resistance in the treatment of ESLD-induced SBP and the need for targeted therapy in the Pakistani population.

\section{Conclusions}

Early and effective antimicrobial treatment is crucial in the management of cirrhotic patients with superimposed bacterial ascites. The choice of an empirical therapy should be based not only on the severity and origin of the infection but also on the local microbiological profile. In the absence of microbiological investigations that demonstrate the presence of gram-negative MDR bacteria, the carbapenems should not be empirically administered unless there is an established risk of MDR bacteria. The administration of inappropriate therapy is associated with increased mortality; hence, the epidemiological patterns of bacterial resistance in our regional hospitals should be evaluated and monitored.

\section{Additional Information \\ Disclosures}

Human subjects: Consent was obtained by all participants in this study. Animal subjects: All authors have confirmed that this study did not involve animal subjects or tissue. Conflicts of interest: In compliance with the ICMJE uniform disclosure form, all authors declare the following: Payment/services info: All authors have declared that no financial support was received from any organization for the submitted work. Financial relationships: All authors have declared that they have no financial relationships at present or within the previous three years with any organizations that might have an interest in the submitted work. Other relationships: All authors have declared that there are no other relationships or activities that could appear to have influenced the submitted work.

\section{References}

1. Biecker E: Diagnosis and therapy of ascites in liver cirrhosis . World J Gastroenterol. 2011, 17:1237-1248. 10.3748/wjg.v17.i10.1237

2. Hurwich DB, Lindor KD, Hay JE, Gross JB Jr, Kaese D, Rakela J: Prevalence of peritonitis and the ascitic fluid protein concentration among chronic liver disease patients. Am J Gastroenterol. 1993, 88:1254-1257.

3. Dever JB, Sheikh MY: Spontaneous bacterial peritonitis-bacteriology, diagnosis, treatment, risk factors and prevention. Aliment Pharmacol Ther. 2015, 41:1116-1131. 10.1111/apt.13172

4. Bibi S, Ahmed W, Arif A, Khan F, Alam SE: Clinical, laboratory and bacterial profile of spontaneous bacterial peritonitis in chronic liver disease patients. J Coll Physicians Surg Pak. 2015, 25:95-99.

5. Nouman S, Hussain A, Hussain M, Ahmed M: Frequency of spontaneous bacterial peritonitis in chronic liver disease. Ann King Edward Med Uni. 2010, 16:112-115.

6. Zaman A, Kareem R, Mahmood R, Hameed K, Khan EM: Frequency of microbial spectrum of spontaneous bacterial peritonitis in established cirrhosis liver. J Ayub Med Coll Abbottabad. 2011, 23:15-17.

7. Koulaouzidis A, Bhat S, Karagiannidis A, Tan WC, Linaker BD: Spontaneous bacterial peritonitis. Postgrad Med J. 2007, 83:379-383. 10.1136/pgmj.2006.056168

8. Koulaouzidis A, Bhat S, Saeed AA: Spontaneous bacterial peritonitis. World J Gastroenterol. 2009, 15:10421049. 10.3748/wig.15.1042

9. Tandon P, Garcia-Tsao G: Bacterial infections, sepsis, and multi-organ failure in cirrhosis. Semin Liver Dis. 2008, 28:26-42. 10.1055/s-2008-1040319

10. Wiest R, Krag A, Gerbes A: Spontaneous bacterial peritonitis: recent guidelines and beyond . Gut. 2012, 61:297-310.

11. Alaniz C, Regal RE: Spontaneous bacterial peritonitis: a review of treatment options .P T. 2009, 34:204-210.

12. Rimola A, Garcia-Tsao G, Navasa M, Piddock LJ, Planas R, Bernard B, Inadomi JM: Diagnosis, treatment and prophylaxis of spontaneous bacterial peritonitis: a consensus document. J Hepatol. 2000, 32:142-153. 
13. Solà, E, Ginès, P: Renal and circulatory dysfunction in cirrhosis: current management and future perspectives. J Hepatol. 2010, 53:1135-1145. 10.1016/j.jhep.2010.08.001

14. Malghani WS, Chaudhary FM, Ilyas M, Ud Din AT, Ud Din AT: Spontaneous bacterial peritonitis (SBP). Spectrum of bacterial flora causing spontaneous bacterial peritonitis (SBP) in patients with liver cirrhosis. Professional Med J. 2018, 25:749-752. 10.29309/TPMJ/18.4465

15. Byass P: The global burden of liver disease: a challenge for methods and for public health . BMC Med. 2014, 12:159. 10.1186/s12916-014-0159-5

16. The global burden of kidney disease and the sustainable development goals . (2018). Accessed: June 29, 2019: https://www.who.int/bulletin/volumes/96/6/17-206441/en/.

17. Fernández J, Acevedo J, Castro M, et al.: Prevalence and risk factors of infections by multi-resistant bacteria in cirrhosis: a prospective study. Hepatology. 2012, 55:1551-1561.

18. Salerno F, Cazzaniga M: Therapeutic strategies and emergence of multiresistant bacterial strains . Intern Emerg Med. 2010, 5:45-51. 10.1007/s11739-010-0447-9

19. Guarner C, Soriano G: Spontaneous bacterial peritonitis. Semin Liver Dis. 1997, 17:203-217. 10.1055/s-20071007198

20. Bhat G, Vandana KE, Bhatia S, Suvarna D, Pai CG: Spontaneous ascitic fluid infection in liver cirrhosis: bacteriological profile and response to antibiotic therapy. Indian J Gastroenterol. 2013, 32:297-301. 10.1007/s12664-013-0329-y

21. Bruns T, Zimmermann HW, Stallmach A: Risk factor sand outcome of bacterial infections in cirrhosis . World J Gastroenterol. 2014, 20:2542-2554. 10.3748/wjg.v20.i10.2542

22. Toshikuni N, Arisawa T, Tsutsumi M: Hepatitis C-related liver cirrhosis - strategies for the prevention of hepatic decompensation, hepatocarcinogenesis, and mortality. World J Gastroenterol. 2014, 20:2876-2887. 10.3748/wjg.v20.i11.2876

23. Ortiz J, Vila MC, Soriano G, et al.: Infections caused by Escherichia coli resistant to norfloxacin in hospitalized cirrhotic patients. Hepatology. 1999, 29:1064-1069. 10.1002/hep.510290406

24. Fernández J, Ruiz del Arbol L, Gómez C, et al.: Norfloxacin vs ceftriaxone in the prophylaxis of infections in patients with advanced cirrhosis and hemorrhage. Gastroenterology. 2006, 131:1049-1056. 10.1053/j.gastro.2006.07.010

25. Ascione T, Di Flumeri G, Boccia G, De Caro F: Infections in patients affected by liver cirrhosis: an update . Infez Med. 2017, 25:91-97.

26. Angeloni S, Leboffe C, Parente A, Venditti M, Giordano A, Merli M, Riggio O: Efficacy of current guidelines for the treatment of spontaneous bacterial peritonitis in the clinical practice. World J Gastroenterol. 2008, 14:2757-2762. 10.3748/wjg.14.2757

27. Piroth L, Pechinot A, Minello A, et al.: Bacterial epidemiology and antimicrobial resistance in ascitic fluid: a 2-year retrospective study. Scand J Infect Dis. 2009, 41:841-847. 10.3109/00365540903244535

28. Lutz P, Nischalke HD, Krämer B, et al.: Frequency of resistance to commonly used antibiotics among a German cohort of patients with spontaneous bacterial peritonitis challenges current treatment recommendations. J Hepatol. 2016, 64:S664. 10.1016/S0168-8278(16)01250-2

29. Joly-Guillou ML, Kempf M, Cavallo JD: Comparative in vitro activity of meropenem, imipenem and piperacillin/tazobactam against 1071 clinical isolates using 2 different methods: a French multicentre study. BMC Infect Dis. 2010, 10:72. 10.1186/1471-2334-10-72 